\title{
Sliding Scale in the Management of Allergic Conjunctivitis
}

\author{
Ahmad Zeeshan Jamil ${ }^{1}$, Muhammad Luqman Ali Bahoo ${ }^{2}$, Zahid Kamal ${ }^{3}$ \\ Muhammad Rizwan ${ }^{4}$, Muhammad Ovais ${ }^{5}$ \\ 1,3,4,5 Sahiwal Medical College, Sahiwal, ${ }^{2}$ CMH Institute of Medical Sciences, Bahawalpur
}

\begin{abstract}
Purpose: To verify the usability of the sliding scale in the management of allergic conjunctivitis.

Study Design: Quasi-Experimental.

Place and Duration of Study: District Headquarter Teaching Hospital/Sahiwal Medical College, Sahiwal, from January 2016 to December 2019.

Methods: A sliding scale was developed and used to score the severity of the ocular allergy. Patients were instructed to use the sliding scale to adjust the treatment regimen and follow-up at regular intervals. At baseline and at third follow-up visit, sliding scale score and use of drug regimens were noted. At third follow-up visit patient's satisfaction and disease control were documented.

Results: There were 398 patients. Mean age of patients was $29.45 \pm 18.77$ years. At baseline, $62.8 \%$ of patients were using topical steroids while at third follow-up visit only $5 \%$ of patients were using topical steroids. Mean interval of patients' visit to the hospital was $8.53 \pm 1.44$ weeks. Non-parametric Mann Whitney test was used to calculate the difference in means of sliding scale scores at baseline $(4.49 \pm 2.39)$ and third follow-up visit $(1.03 \pm$ 1.68). $Z$ score value was -16.917 and significance was 0.000 . For patient's satisfaction at third follow-up visit Chisquare value was 263.759 with significance value of 0.000 . For disease control at third follow-up visit chi-square value was 223.123 with significance value of 0.000 .
\end{abstract}

Conclusion: Use of sliding scale in the management of allergic conjunctivitis was well accepted by the patients. It helped in disease control with minimal use of topical steroids and less frequent visits to the hospital.

Key Words: Allergic Conjunctivitis, Steroids, Trantas dots.

How to Cite this Article: Jamil AZ, Bahoo MLQ, Kamal Z, Rizwan M, Ovais M. Sliding Scale in the Management of Allergic Conjunctivitis. Pak J Ophthalmol. 2020; 36 (4): 365-370.

Doi: https://doi.org/10.36351/pjo.v36i4.1055

\section{INTRODUCTION}

Allergic diseases have increased over the last one decade. Ophthalmologists frequently encounter ocular allergy patients. Air pollution and genetic

Correspondence: Muhammad Luqman Ali Bahoo

Department of Ophthalmology, Sahiwal Medical College, Sahiwal

Email: drluqmanali@yahoo.com

Received: April 22, 2020

Accepted: July 29, 2020 predisposition are considered responsible. ${ }^{1}$ Moreover, climate has changed due to industrialization and urbanization. The rise in environmental temperatures and intense seasons can lead to earlier pollen season and subsequently more ocular allergies. Allergic conjunctivitis disease (ACD) is increasing worldwide. ${ }^{2}$

The term allergic conjunctivitis (AC) or ocular allergy (OA) refers to a collection of ocular surface disorders that affect the palpebral and bulbar conjunctiva. ${ }^{3}$ Conjunctiva is the mucous membrane of the eye surface and it is persistently and commonly exposed to extensively diverse antigens present in air. ${ }^{4}$ In allergic conjunctivitis (AC) $\operatorname{IgE}$ and non- $\operatorname{IgE}$ 
mediated hypersensitivity reactions take place. $^{5}$ Resulting inflammation leads to pathological interactions between different immune cells and local cells of eye resulting in various lesions on conjunctiva. Itching is the hall mark of allergic conjunctivitis. Other clinical presentations include conjunctival hyperemia, photophobia, watering and conjunctival and lid swelling. ${ }^{6}$ Almost $40 \%$ of the population is affected by symptoms of AC. Among the patients of ACD, $90-$ 95\% cases are of seasonal allergic conjunctivitis (SAC) or perennial allergic conjunctivitis (PAC). ${ }^{7}$ The intense sign and symptoms of disease are so troublesome that these can lead to reduction in work efficiency, absence from educational institutes and work place, restricted daily activities and poor quality of life. ${ }^{8}$

Among different drugs, the anti-allergic eye drops are the first drug of choice for basic treatment of the disease, followed by selective use of steroid eye drops depending upon the severity of the disease. In case of more severe disease immunosuppressive eye drops, oral steroids, supratarsal steroid injection and surgical treatment such as papillary resection can also be considered in addition to anti-allergic and steroid eye drops. While managing $\mathrm{AC}$, the sufferers of $\mathrm{AC}$ face an economic burden, which is imposed on them by medication and health care visits as well as decreased productivity. The appropriate management of AC advocates comprehensive strategies like avoidance of suspected allergens, relief of symptoms and suppression of inflammatory response with pharmacological preparations. ${ }^{9}$ For assessing the clinical severity of ACD, translating clinical observations into quantitative clinical scores is expedient. Multiple studies measured and reported the effects of therapeutic drugs using clinical scores. ${ }^{10,11}$

Due to consumption of health care resources and reduced quality of life of affected patients of $\mathrm{AC}$, the studies on different prospective of the disease are justified. In an attempt to minimize the burden of this commonly prevalent disease on the health care resources, a sliding scale was designed and used to score the severity of ocular allergy with an additional aim to educate the patients to use sliding scale for adjustment of the prescribed treatment regimen at home themselves. This study was carried out to authenticate the usability of sliding scale in the management of allergic conjunctivitis. The sliding scale make it possible to tangibly determine the indication or cancellation stage for therapeutic drugs by the patient himself. Along with it, sharing of such type of sliding scale enhances the doctor patient bonding and vigilant involvement of patient in mutual scheduling for treatment and follow-up. It might also boost sense of self-care in patients as well.

\section{METHODS}

This quasi-experimental study was conducted in District Headquarter Teaching Hospital affiliated with Sahiwal Medical College, Sahiwal. Duration of study was from January 2016 to December 2019. Study was approved by the institutional review board. Informed consent was obtained from all the participants and parents in case of minors. Non-probability purposive sampling technique was used. Sample size was calculated according to the following formula:

$\mathrm{S}=\mathrm{Z}^{2} \mathrm{p}(1-\mathrm{p}) / \mathrm{M}^{2}$

$\mathrm{S}$ is the sample size

$\mathrm{Z}$ is $\mathrm{Z}$ score its value is 1.96

$\mathrm{P}$ is the population proportion assumed to be $50 \%$ or 0.5

$\mathrm{M}$ is the margin of error that is taken $5 \%$ or 0.05

$\mathrm{S}=(1.96)^{2}(0.5)(1-0.5) /(0.05)^{2}$

$=384.16$

$=384$

Inclusion criteria was patients older than 2 years, bilateral disease. Exclusion criteria was steroid responders, patients of glaucoma, trauma, intraocular inflammation, diabetic retinopathy, age related maculopathy, dry eyes and with history of ocular surgery in the previous six months. Failure to come on follow-up visits and failure to follow treatment according to sliding scale also resulted in exclusion of patient from the study.

Diagnosis of allergic conjunctivitis was based on history and clinical examination. History of nasal allergy, atopy, pollen allergy, dust allergy, seasonal exacerbation, respiratory allergy, skin allergy and previous treatment were taken into account. Patients or parents of minors were asked for symptoms of itching, redness of conjunctiva and photophobia. Thorough clinical examination was performed. Presence of papillae, Horner's Trantas dots, severity of redness of conjunctive, type of discharge from eyes and severity of photophobia was noted. 
A sliding scale was developed whereby clinical presentations of itching, redness of conjunctiva and photophobia were used to calculate score of sliding scale. Ophthalmologists calculated score of sliding scale at examination visits and patients used that scale at home. Score of 4 was given in case of continuous itching (present all the time), score of 3 was given in case of frequent itching (present $50 \%$ of time), score of 2 was given in case of occasional episode of severe itching (present $25 \%$ of time), score of 1 was given in case of occasional episode of mild itching (present $25 \%$ of time) and score of 0 was given in case of absence of itching.

Likewise score of 3 was given in case of complete bulbar conjunctival redness, score of 2 was given in case of redness involving less than full and more than half of the bulbar conjunctiva. Score of 1 was given in case of redness involving less than half of the bulbar conjunctiva. Score of 0 was given in case of absence of bulbar conjunctival redness.

Score of 3 was given in case where history suggests severe photophobia (present in dim light). Score of 2 was given in case of moderate photophobia (present in well-lit room). Score of 1 was given in case of mild photophobia (present in daylight). Score of 0 was given in case of absence of photophobia in daylight.

Patients/parents in case of minors were instructed how to use sliding scale to calculate score of their allergy severity and use of medicines accordingly. Patients reviewed their score after every one week and adjusted treatment. Follow-up of patients was according to the schedule of sliding scale. For example, on baseline examination, a score of 5 was noted and patient started treatment accordingly. One week later patient reviewed his symptoms and scored the severity of his disease. This time a score of 3 was noted and patients modified treatment according to the new score. In that way, patients reviewed their symptoms every week and managed their treatment up or down the sliding scale. Content validity of the sliding scale was done with the help of three ophthalmologists to approve the selection of scale. A pilot study was conducted before the full-scale study to find out the feasibility. English and Urdu version of the patient's information sheet and sliding scale were developed.

After first examination, patients were followed up for three consecutive visits. Patient's satisfaction at third follow-up examination and treatment success was defined by sliding scale score of 3 or less at third follow-up examination.

For statistical analysis, statistical package for social sciences (SPSS) version 23 was used. Frequencies were calculated for gender, involvement of palpebral and bulbar conjunctiva, patient's satisfaction and treatment success. Mean and standard deviation was calculated for age, sliding scale score at baseline, first follow-up, second follow-up and third follow-up. Mean and standard deviation was also calculated for interval of first, second and third followup visits.

Non-parametric Mann Whitney test was used to calculate difference in means of sliding scale at baseline and at third follow-up visit. $p$ value less than 0.05 was considered statistically significant.

For patient's satisfaction and treatment success at third follow-up visit Chi square test was used with a pvalue of less than 0.05 .

\section{RESULTS}

There were 398 patients. Mean age of the patients was $29.45 \pm 18.77$ years. Age range was from 3 years to 64 years. There were $192(48.2 \%)$ male and $206(51.8 \%)$ female patients. In $278(69.8 \%)$ cases palpebral conjunctiva was involved, in $58(14.6 \%)$ patients limbal papillary conjunctivitis was present and in 62 $(15.6 \%)$ cases both palpebral and limbal papilla were present at baseline visit. Frequencies of itching, conjunctival redness and photophobia at baseline visit is given in Chart number 1. By the use of sliding scale $361(90.7 \%)$ patients were satisfied while 37 (9.3\%) patients were not satisfied at third follow-up visit. In $348(87.4 \%)$ cases, allergic conjunctivitis was controlled at third follow-up visit. In $50(12.6 \%)$ cases allergic conjunctivitis was not controlled at third follow-up visit. Sliding scale score mean and standard deviation is given in table number 1. Examination visit duration mean and standard deviation is given in table number 2 .

Table 1: Sliding Scale Score.

\begin{tabular}{lc}
\hline Examination Visit & $\begin{array}{c}\text { Score Mean \& Standard } \\
\text { Deviation }\end{array}$ \\
\hline Baseline & $4.49 \pm 2.39$ \\
First follow-up & $2.81 \pm 2.17$ \\
Second follow-up & $1.38 \pm 1.77$ \\
Third follow-up & $1.03 \pm 1.68$ \\
\hline
\end{tabular}


Table 2: Follow-up Interval from Baseline Examination.

\begin{tabular}{lc}
\hline Follow-up Visit & Interval (Weeks) \\
\hline First & $5.96 \pm 2.87$ \\
Second & $7.64 \pm 2.11$ \\
Third & $8.53 \pm 1.44$ \\
\hline
\end{tabular}

At initial visit, $62.8 \%$ patients were started on topical steroids while at third follow-up visit 5\% of patients were using topical steroids. Eight percent of patients were using oral anti-allergy medicine at baseline examination while $5 \%$ of patients were using oral anti-allergy medicine at third follow-up visit. At baseline examination, $70.4 \%$ of patients were using cyclosporine eye drops while at third follow-up examination $5 \%$ of patients were using cyclosporine eye drops. At baseline examination, all patients were using olopatadine eye drops while at third follow-up examination, only $12.6 \%$ patients were using

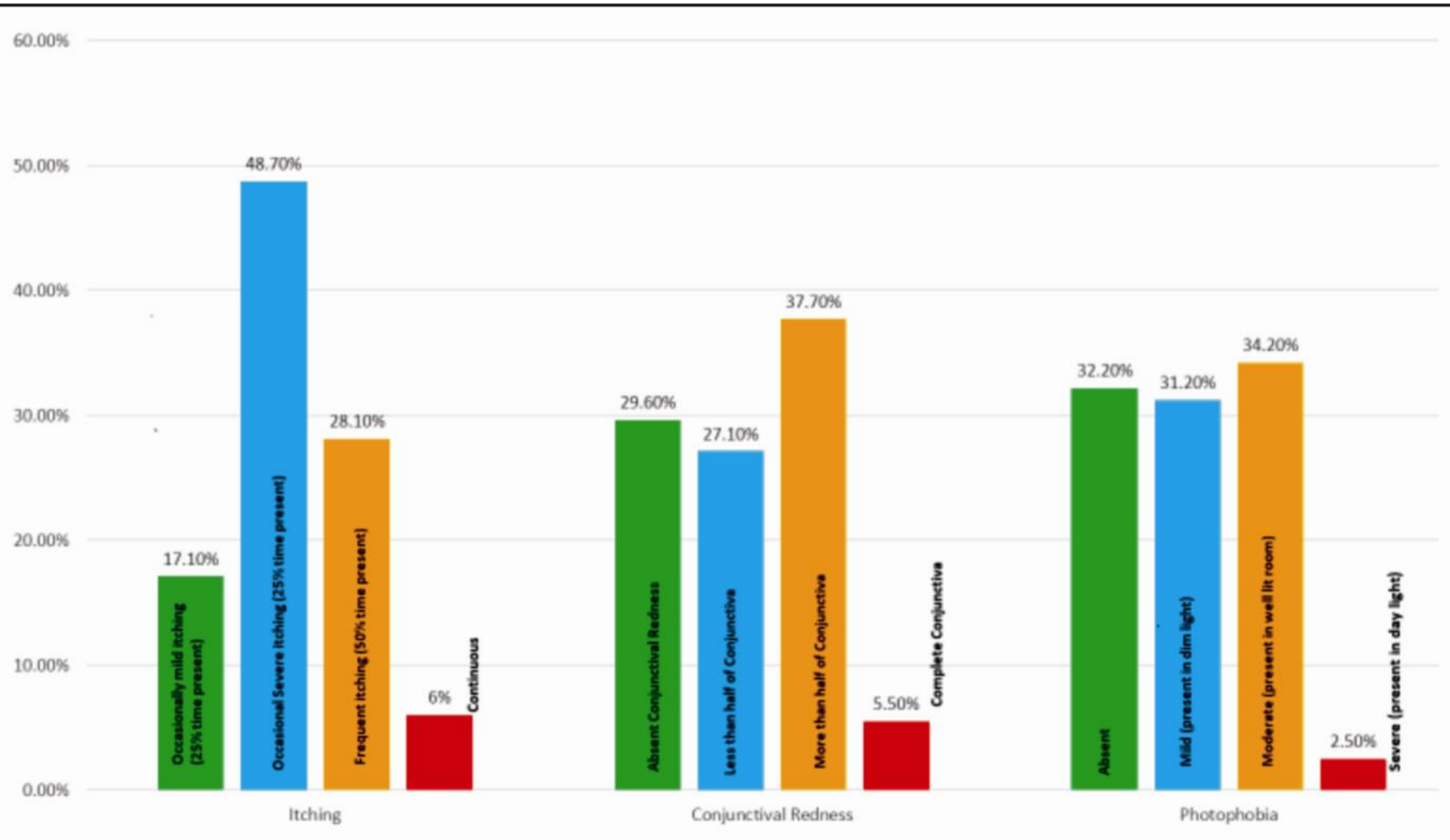

Chart 1: Frequencies of Itching, Conjunctival Redness and Photophobia at Baseline Visit.

Table 3: Drug Regimen at Baseline and Third Follow-up.

\begin{tabular}{|c|c|c|}
\hline Drug Regimen & Baseline & $\begin{array}{c}\text { Third } \\
\text { Follow-up }\end{array}$ \\
\hline (0) No Drugs & 0 & $174(43.7 \%)$ \\
\hline (1) Olopatadine ${ }^{\alpha}$ once a day & $48(12.1 \%)$ & $174(43.7 \%)$ \\
\hline (2) Olopatadine twice a day & $70(17.6 \%)$ & $20(5 \%)$ \\
\hline (3) $2+$ Cyclosporine $^{\otimes}$ & $30(7.5 \%)$ & $10(2.5 \%)$ \\
\hline (4) $3+$ One steroid $^{\beta}$ drop Once a week & $44(11.1)$ & 0 \\
\hline (5) $3+$ One steroid drop after two days & $60(15.1)$ & 0 \\
\hline (6) $3+$ One steroid drop once a day & $44(11.1)$ & 0 \\
\hline (7) 3+ One steroid drop Twice a day & $70(17.6)$ & $10(2.5 \%)$ \\
\hline $\begin{array}{l}\text { (8) } 3+\text { One steroid drop Thrice a day } \\
+ \text { Oral Desloratadine } \\
\delta\end{array}$ & $18(4.5)$ & $10(2.5 \%)$ \\
\hline $\begin{array}{l}\text { (9) } 3+\text { One steroid drop Four times in } \\
\text { a day+ Oral Desloratadine }+ \text { steroid }\end{array}$ & $4(1.0)$ & 0 \\
\hline
\end{tabular}

(10) $3+$ One steroid drop Six times in a day + Oral Desloratadine + ointment $10(2.5) \quad 0$ at night

\section{Key}

$\otimes=$ Cyclosporine Eye Drops $0.05 \%$

$\alpha=$ Olopatadine Eye Drops $0.2 \%$

$\beta=$ Flouromethalone $0.25 \%$ Eye Drops

$\delta=$ Desloratadine Tablet $5 \mathrm{mg}$ or Desloratadine Syrup 0.5 $\mathrm{mg} / \mathrm{ml}$

$\varepsilon=$ Flouromethalone $0.1 \%$ Eye Ointment

olopatadine eye drops. Table number 3 shows drug regimen at baseline and at third follow-up visit. 
Nonparametric Mann Whitney test was used to calculate difference in means of sliding scale at baseline and third follow-up visit. Z score value was 16.917 and significance was 0.000 .

For patient's satisfaction at third follow-up visit Chi-square test was used. Chi-square value was 263.759 with a significance value of 0.000 . For disease control at third follow-up visit chi-square value was 223.123 with a significance value of 0.000 .

\section{DISCUSSION}

In our study mean age of the patients was $29.45 \pm$ 18.77 years. Age range was from 3 years to 64 years. Allergic conjunctivitis is more prevalent in children. A study conducted among 818 children of age 5-19 years in Karachi found that $19.2 \%$ had allergic conjunctivitis with significant association between increasing age and allergic conjunctivitis. ${ }^{12}$ Review of literature shows different ocular allergies affect different age groups. Seasonal allergic conjunctivitis most frequently affects individuals younger than 20 years, vernal keratoconjunctivitis is most often seen in patients younger than 10 years, atopic keratoconjunctivitis is most frequently encountered in persons from 30 to 50 years of age and giant papillary conjunctivitis is seen is teenagers and young adults. ${ }^{13}$

Ocular allergy may be associated with allergies of nose, skin or respiratory system. ${ }^{14,15}$ Treatment of systemic allergy often ameliorates the clinical presentation of ocular allergy. Systemic anti-allergy medicine proved helpful in severe cases of ocular allergies without involvement of other parts of the body. ${ }^{16}$ In the present study oral anti-allergic medicine was started in $8 \%$ of patients with good results.

For the treatment of ocular allergies, topical steroids are reserved for acute exacerbations and in cases that are not controlled otherwise. For the treatment of ocular allergies steroids are used topically, sub conjunctively, supratarsally, orally and nasally. ${ }^{17}$ Steroid use may be associated with corneal infection, raised intraocular pressure and formation of cataract. ${ }^{18}$ Judicious use of steroids is mandatory considering its adverse effects. Allergic conjunctivitis can significantly affect the quality of life, result in economic and educational loss. Eye rubbing associated with ocular allergy can cause other eye problems like progression of myopia and development of Keratoconus. ${ }^{19}$
Shoji and co-authors developed a scoring system to grade different types of allergic conjunctivitis into mild, moderate and severe categories. ${ }^{20}$ Our grading system is different from the grading system used by Shoji and co-authors. The clinical features used by our patients were supervised by the ophthalmologists to decide a treatment regimen.

Sliding scale use results in follow-up visits at longer intervals and use of minimal eye drops for the control of Thereby it is possible to avoid unnecessary use of steroids. At the same time, an appropriate dose of medicine allows adequate control of the disease. ${ }^{21}$ Moreover, better control of ocular allergy results in minimal use of medicines and less frequent follow-up visits to hospital hence, saving time and money of the patients. ${ }^{1}$

Limitation our study was that duration of our follow-up visits was small. We did not separately identify patients of seasonal allergic conjunctivitis, Vernal keratoconjunctivitis and atopic conjunctivitis. Individual variation in the perception of symptoms exists. Selection of drugs in different regimens was authors' choice. Different drugs can be compared in future for their effects using proposed sliding scale.

Nevertheless, our proposed sliding scale was useful in the management of allergic conjunctivitis whereby patients were actively involved in planning a drug regimen and follow-up visit under the supervision of the treating ophthalmologist. Minimal use of topical steroids with increasing interval between follow-up visits was associated with improved patient's satisfaction and more disease control at the end of the study.

\section{CONCLUSION}

Use of sliding scale in the management of allergic conjunctivitis was well accepted by the patients. It helped in disease control with minimal use of topical steroids and less frequent visits to the hospital.

\section{Ethical Approval}

The study was approved by the Institutional review board/Ethical review board.

\section{Conflict of Interest}

Authors declared no conflict of interest. 


\section{REFERENCES}

1. Kohli CM, Kohli GM. Assessment of clinical profile of patients with allergic conjunctivitis. J Adv Med Dent Scie Res. 2014; 2 (4): 190-193.

2. Gomes PJ. Trends in prevalence and treatment of ocular allergy. Curr Opin Allergy Clin Immunol. 2014; 14: 451-456.

3. Leonardi A, Bogacka E, Fauquert JL, Kowalski ML, Groblewska A, Jedrzejczak-Czechowicz M, et al. Ocular allergy: recognizing and diagnosing hypersensitivity disorders of the ocular surface. Allergy, 2012; 67: 1327-1337.

4. Cronau H, Kankanala RR, Mauger T. Diagnosis and management of red eye in primary care. Am Fam Physician, 2010; 81 (2): 137-144.

5. Takamura E, Uchio E, Ebihara N, Ohno S, Ohashi Y, Okamoto S, et al. Japanese guidelines for allergic conjunctival diseases 2017. Allergol Int. 2017; 66 (2): 220-229.

6. Azari AA, Barney NP. Conjunctivitis: A systematic review of diagnosis and treatment. JAMA. 2013; 310 (16): 1721-1729. Doi: 10.1001/jama.2013.280318.

7. Singh K, Axelrod S, Bielory L. The epidemiology of ocular and nasal allergy in the United States, 19881994. J Allergy Clin Immunol. 2010; 126: 778-783.

8. Chigbu DI. The pathophysiology of ocular allergy: A review. Cont Lens Anterior Eye, 2009; 32 (1): 3-15; Quiz 43-44.

9. O'Brien TP. Allergic conjunctivitis: An update on diagnosis and management. Curr Opin Allergy Clin Immunol. 2013; 13 (5): 543-549.

10. Shoji J, Inada N, Sawa M. Evaluation of novel scoring system named 5-5-5 exacerbation grading scale for allergic conjunctivitis disease. Allergol Int. 2009; 58 (4): 591-597. Doi: 10.2332/allergolint.09-OA-0100. Epub 2009 Sep 25.

11. Bielory L, Meltzer EO, Nichols KK, Melton R, Thomas RK, Bartlett JD. An algorithm for the management of allergic conjunctivitis. Allergy Asthma Proc. 2013; 34 (5): 408-420.

Doi: 10.2500/app.2013.34.3695.

12. Baig R, Ali AW, Ali T, Ali A, Shah MN, Sarfaraz A, et al. Prevalence of allergic conjunctivitis in school children of Karachi. J Pak Med Assoc. 2010; 60 (5): 371-373.

13. Baab S, Le PH, Kinzer EE. Allergic Conjunctivitis. [Updated 2020 Feb 21]. In: Stat Pearls. Treasure Island (FL): Stat Pearls Publishing; 2020 Jan-. Available from: https://www.ncbi.nlm.nih.gov/books/NBK448118/

14. Mashige KP. Ocular allergy. Health SA Gesondheid. 2017; 22: 112-122.
15. Maspero J, Lee BW, Katelaris CH, Potter PC, Cingi C, Lopatin A, et al. Quality of life and control of allergic rhinitis in patients from regions beyond western Europe and the United States. Clin Exp Allergy, 2012; 42 (12): 1684- 1696.

Doi: $10.1111 / \mathrm{j} .1365-2222.2012 .04025 \mathrm{x}$.

16. Leonardi A. Management of vernal keratoconjunctivitis. Ophthalmol Ther. 2013; 2 (2): 73 88.

17. Ackerman S, Smith LM, Gomes PJ. Ocular itch associated with allergic conjunctivitis: latest evidence and clinical management. Ther Adv Chronic Dis. 2016; 7 (1): 52-67.

18. Bowling E. The conjunctivitis conundrum. Review of Optometry, 2020 February 15. Available from: https://www.reviewofoptometry.com/article/theconjunctivitis-conundrum. (Accessed March 22, 2020)

19. Pitt AD, Smith AF, Lindsell L, Voon LW, Rose PW, Bron AJ. Economic and quality-of-life impact of seasonal allergic conjunctivitis in Oxfordshire. Ophth Epidemiol. 2004; 11 (1): 17-33.

20. Shoji J, Inada N, Sawa M. Evaluation of novel scoring system named 5-5-5 exacerbation grading scale for allergic conjunctivitis disease. Allergol Int. 2009 Dec; 58 (4): 591-7. Doi: 10.2332/allergolint.09-OA-0100. Epub 2009 Sep 25. PMID: 19776677.

21. Rathi VM, Murthy SI. Allergic conjunctivitis. Community Eye Health, 2017; 30 (99): S7-S10.

\section{Authors' Designation and Contribution}

Ahmad Zeeshan Jamil; Associate Professor: Concepts, Design, Data Analysis, Manuscript preparation.

Muhammad Luqman Ali Bahoo; Associate Professor and Head of Department: Literature research, Manuscript preparation.

Zahid Kamal; Professor and Head of Department: Manuscript preparation, Manuscript editing, Manuscript review.

Muhammad Rizwan; Assistant Professor: Data acquisition, Statistical Analysis, Manuscript preparation.

Muhammad Ovais; Senior Registrar: Literature research, Data Analysis, Manuscript preparation. 